\title{
Evolving Concepts of Skin and Mucosal Biopsy in Facial Vascularized Composite Allotransplantation
}

\author{
Michael Sosin • Jhade D. Woodall • Benjamin D. Schultz • \\ Arif Chaudhry • Branko Bojovic • Michael R. Christy • \\ Eduardo D. Rodriguez • Cinthia B. Drachenberg
}

Published online: 18 June 2014

(C) Springer International Publishing AG 2014

\begin{abstract}
Facial vascularized composite allotransplantation has ushered in a new era in treating complex facial injuries that cannot be reconstructed using traditional techniques. Multiple teams have reported their experiences in monitoring for allograft rejection using skin and mucosal biopsies. The association of biopsy findings and clinical observations are poorly understood and are continuously being redefined. We review the world's experience in monitoring skin and mucosal histological findings in facial transplantation, review acute rejection, antibody-mediated rejection, chronic rejection, and describe our institutional experience in the monitoring and management of facial allograft histology.
\end{abstract}

Keywords Face transplant - Vascularized composite allotransplantation $\cdot$ Skin $\cdot$ Mucosa $\cdot$ Skin biopsy $\cdot$ Rejection

M. Sosin • B. D. Schultz • B. Bojovic · M. R. Christy

Division of Plastic, Reconstructive, and Maxillofacial Surgery,

R Adams Cowley Shock Trauma Center, 110 South Paca Street,

Room 3-N-146, Baltimore, MD 21201, USA

M. Sosin

e-mail: sosinmi@gmail.com

B. D. Schultz

e-mail: Benjamin.schultz@som.umaryland.edu

B. Bojovic

e-mail: bbojovic@umm.edu

M. R. Christy

e-mail: mchristy@umm.edu

A. Chaudhry • E. D. Rodriguez

Department of Plastic Surgery, New York University Langone

Medical Center, 305 East 33rd Street, New York, NY 10016, USA
A. Chaudhry
e-mail: dr.a.chaudhry@gmail.com
E. D. Rodriguez
e-mail: eduardo.rodriguez@nyumc.org

\section{Introduction}

The early 21 st century has ushered in the expanding field of vascularized composite allotransplantation (VCA), which has evolved into a clinical reality. To date, a total of 101 hand transplants and 28 face transplants have been performed worldwide [1]. Although mechanisms of immunoregulation and tolerance in solid organ transplantation and VCA overlap, there are inherent differences in the biology of allotransplantation that have been elucidated in animal models and human clinical experience. An amalgam of clinical data has been and continues to be reported from multiple institutions regarding their observations and analysis of the immune response to facial and limb VCA. Facial transplantation improves quality of life from a functional, nutritional, emotional, and social

\author{
C. B. Drachenberg $(\bowtie)$ \\ Department of Pathology, University of Maryland Medical Center, \\ Baltimore, MD, USA \\ e-mail: cdrac001@umaryland.edu
}

J. D. Woodall

Department of Surgery, University of Maryland Medical Center, 685 W. Baltimore St., Suite 457A, Baltimore, MD 21201, USA

e-mail: Jhade.woodall@gmail.com

\section{E. D. Rodriguez}

Department of Plastic Surgery,

New York University Langone Medical Center,

305 East 33rd Street,

New York, NY 10016, USA

C. B. Drachenberg

University of Maryland School of Medicine,

University of Maryland Hospital, 22 South Greene St.,

Baltimore, MD 21201, USA 
perspective in patients that have complex facial defects not amenable to traditional reconstructive techniques $[2,3]$. Nevertheless, facial VCA requires lifelong immunosuppression, which ultimately limits its broader clinical application. A better understanding of the mechanisms involved in rejection or improvement in immunosuppressive drug therapies will expand the field of reconstructive transplantation.

Facial allograft monitoring is largely based upon skin and mucosal biopsies. Currently, the implications and association of biopsy findings with clinical observations remain poorly understood. The purpose of this manuscript is to review the experience of skin and mucosal biopsies in facial transplantation, review rejection, and describe our institutional experience following facial VCA.

\section{History}

The success of the first human hand transplant in 1998 and eventually the first human face transplant in 2005 were a direct result of translational research in small and large animal studies $[4,5]$. The implementation of novel and effective immunosuppressive protocols established in solid organ transplantation provided the foundation to attempt what is still considered to be an experimental procedure. In addition to reviewing other clinical experiences with skin and mucosal histological interpretation, we highlight the critical concepts gained from our experience in facial transplantation [6] and in our assessment of allograft rejection.

\section{Acute Rejection}

It is still unclear whether skin is the main target of acute rejection, but most clinical VCA experiences show that signs of acute rejection manifest in the skin. Aside from the allograft skin, most of the other tissue components comprising the allograft are not commonly obtained for histological evaluation leaving a paucity of clinical data regarding muscle, tendon, cartilage, and bone as potential targets for rejection. However, even during severe rejection the deeper tissue seems to be less involved relative to the skin [7].

Acute rejection is graded using the Banff 2007 guidelines [8]. Microscopic characteristics often demonstrate nonspecific changes in the dermis and sometimes the epidermis. Early evidence of rejection is seen with perivascular lymphocytic infiltrates in the dermis. The predominant cells are $\mathrm{CD} 3+/$ CD4+ T-cells with occasional CD8+ cytotoxic T-cells, FoxP3+ T-regulatory cells, and CD68+ histiomonocytic cells of recipient origin. Rejection from the dermis will progress to the epidermis and then to the hypodermis if left untreated. As the severity of rejection increases, epidermal findings will include keratinocyte apoptosis, necrosis and vacuolization.
Evolving chronic changes appear as acanthosis, hyperkeratosis, and obliteration of hair follicles and sweat glands [9-11].

Acute rejection is a T-cell mediated response, which is the main target of triple immunosuppressive therapy in VCA (mycophenolate mofetil, tacrolimus, and corticosteroids). This likely explains why acute rejection can be managed if detected in a timely manner. According to the International Hand and Composite Tissue Transplantation Registry, 54.5\% of face allografts experience acute rejection within the first post-transplant year [12]. All patients that received facial transplants developed at least one episode of rejection after greater than one year follow-up [3]. Perhaps the ease of obtaining skin biopsies versus the technical difficulties of biopsying solid organs explains why VCA is associated with a higher incidence of rejection than in solid organ transplantation.

\section{Biopsy of Skin and Mucosa}

Vascularized composite tissue is unique in that multiple tissue types are transplanted including skin, subcutaneous tissue, muscle, tendon, cartilage, mucosa, blood vessels, and bone. Skin is considered the most antigenic tissue [13]. Interestingly, in the setting of VCA, it is less immunogenic than isolated tissue components that are transplanted alone [7]. This finding in animal models seems to be supported in the clinical experience of human VCA. However, the transfer of heterogeneous tissue complicates the process of monitoring allograft rejection. Because the skin is the easiest tissue component to access, is easily visible on clinical examination, rapidly heals without severe functional impairment, and is the most sensitive in manifesting immunologic activity, it is the standard tissue used for monitoring allograft rejection. As a result, our understanding of acute rejection is limited to the skin.

The skin maintains a large burden of antigen presenting cells (Langerhans cells and dendritic cells). Keratinocytes are able to express MHC class II molecules upon activation, and are capable of secreting chemokines to attract large numbers of lymphocytes toward the epidermis [14-17]. This supports the notion of skin being the initial target of acute rejection. In facial transplantation, the allograft skin is traditionally the site of routine biopsies, but multiple groups have reported using oral mucosa or a distant sentinel skin graft in evaluating for subclinical acute rejection.

Interpretation of histologic mucosal findings remain controversial, and there is a lack of consensus as to how to treat the patient based on such findings, especially when they are not consistent with a concurrent skin biopsy or with the clinical presentation. Mucosal biopsies are reported to exhibit higher grades of acute rejection and may also show nonspecific inflammatory patterns [18]. Dubernard et al., in France utilized three potential biopsy sites: allograft skin, oral 
mucosa, and a sentinel skin graft in the inframammary location [19]. To date, sentinel skin grafts have been described in at least five facial transplants $[5,20,21]$. Similar clinical findings with dermatitis or rosacea of the facial allograft may be mistaken for acute rejection, and the sentinel skin graft has added important information for the diagnosis of acute rejection [22••]. Pomahac et al., report more accuracy in using the sentinel skin graft as a means of confirmation for clinical signs of rejection [22••]. Kanitakis et al., described acute rejection patterns of erythema and edema in all sites, but rejection was histologically more severe in the mucosa. Pathologically, 17 of 20 mucosal biopsies were found to show rejection, two of four allograft skin biopsies showed rejection, and four of 11 sentinel skin graft biopsies showed rejection. Clinically the patient was reported to have two episodes of rejection [18]. In Cleveland, Bergfeld et al., described a fouryear experience of facial transplantation biopsies of skin and mucosa. Findings of interface mucositis, sparing the submucosa was common, and increased severity of rejection was also evident. Histologically, 22 of 45 mucosal biopsies showed Grade 2 rejection and nine of 45 showed Grade 3 rejection. In total, 58 of 120 skin biopsies (48 \%) had histologic changes that were assessed as acute rejection. Only 18 pairs of skin and mucosal biopsies were concordant with one another, and 24 pairs of skin and mucosal biopsies revealed Grade 2 or 3 mucosal rejection and Grade 0 or 1 skin rejection. Due to the discrepancy of histologic findings, the patient was treated for rejection when clinical signs were present, which was documented as two episodes [23••, 24].

Complicating matters further, dermatoses and skin lesions may mimic acute rejection as alluded to previously. In the setting of immunosuppression, patients are predisposed to opportunistic infections including but not limited to cytomegalovirus (CMV), Epstein-Barr virus (EBV), and herpes simplex virus (HSV) [25]. They may present clinically as papules, discoloration, and painful lesions. The French group reported early detection of herpetic skin lesions that were treated with decreasing immunosuppression with concomitant topical and oral acyclovir. The same patient later developed molloscum contagiosum along the facial allograft, which was treated with curettage [18]. A thorough profile of both donor and recipient viral status is of paramount importance to accurately diagnose and treat similar outbreaks. A high incidence of CMV infection in hand VCA transplantation [26] and renal transplantation $[27,28]$ has been implicated in triggering an immune response to the allograft and decreased graft survival. This high incidence might be related to a larger viral load capacity in skin endothelium relative to endothelial cells of the kidney, liver, or heart allografts. However, attributing rejection to CMV seropositivity is problematic because the treatment of CMV viremia involves decreasing immunosuppression, which may itself predispose the patient to rejection. In facial VCA there are at least nine documented cases of CMV mismatch between donor and recipient of which all cases report seropositivity in the donor [2, 22••, 29-33]. Although described in upper extremity VCA [17], an association between increased episodes of rejection and CMV mismatch has yet to be correlated in facial transplantation.

In addition to viral pathogens, contact dermatitis, psoriasis, and atopic dermatitis may share similar molecular and cellular mechanisms with acute rejection. Activation of the innate immune system, CD4+/CD8+ T-cells, and stimulation of cytokine release from Langerhans cells and T-cells may initiate a response similar to rejection or potentially induce an episode of acute rejection. It remains unclear whether the overlap of multiple pathogenic processes is mistaken for acute rejection, or if multiple insults to the allograft can spur a subclinical episode of acute rejection. In the absence of clinical rejection, suspicious skin biopsies may be a harbinger of future rejection, which may prompt careful follow-up and early detection of acute rejection.

Uncertainty of inflammatory findings in mucosal samples pervades the VCA community. Bergfeld et al., proposed a grading system of mucosal inflammation in an attempt to correlate it with the Banff 2007 guidelines for skin of VCA $[23 \cdot \bullet]$. Their experience demonstrated rejection to be more severe in the mucosa relative to skin, and they were unable to find any association with clinical signs of rejection. However, they reported that within the first post-transplant year all Grade 3 mucosal rejection was preceded by CMV viremia. Interestingly, this was no longer evident in the second year post-transplant. Kanitakis et al., described multiple episodes of rejection in the oral mucosa that did not correspond to skin biopsies at most time points, but during a clinical episode of rejection the oral mucosa histology showed a more severe inflammatory response [18]. Although a causal clinicopathologic relationship between the patient's HSV mucocutaneous infection and the subsequent development of rejection could not be proven, the authors supported this possibility [18]. Long term follow-up in the same patient at 3-years and 5years showed Grade 0 inflammation of the sentinel skin graft and the oral mucosa. The 4-year biopsies of both sites were found to have Grade 2 rejection in the absence of clinical signs. No treatment was pursued [34]. The Boston group reported mucosal rejection patterns more severe than skin allograft biopsies. Sentinel skin grafting was avoided due to the authors ability to biopsy the allograft without compromising facial aesthetics and based on potential differences of skin antigen presenting cells in different anatomical sites $[22 \bullet \bullet, 35]$. The French group reported a valgancyclovirresistant CMV viremia that coincided with an episode of rejection, which was strongly implicated by the authors as the impetus for rejection [29]. Despite many groups still obtaining mucosal biopsies, in facial transplantation mucosal biopsies are in general not considered a reliable method of detecting acute rejection. 
Considering the possibility of drug toxicity and other side effects leading to mucosal changes is important in patients undergoing facial transplantation because the immunosuppression levels constantly fluctuate based upon patient factors. Medications such as corticosteroids and mycofenolate mofetil are associated with diminishing protective mechanisms of the various mucosal lining in the body. As such, patients may be prone to mucosal erosion and subsequent inflammation throughout the entire orogastrointestinal tract [36, 37]. Furthermore, sirolimus is a common mTOR used in patients that have tacrolimus induced kidney injury and has been shown to cause mucosal ulcers [38, 39]. Complicating matters further, the mucosa is also routinely exposed to a milieu of antigens and foreign bodies. A stimulated inflammatory response, albeit subclinical, may lead to misinterpretation of mucosal histology representing acute rejection.

\section{Experience at the University of Maryland}

Following Institutional Review Board approval at the University of Maryland Medical Center/R. Adams Cowley Shock Trauma Center a multidisciplinary team was led by Eduardo D. Rodriguez M.D., D.D.S. to perform the most extensive, full face transplant to reconstruct the middle and lower facial segments in March of 2012 [6]. A multidisciplinary team approach involving the plastics, transplant, pathology, psychiatry, nephrology, infectious disease, and rehabilitation departments enables a global ability to monitor the progress of the VCA patient. This cohesive approach among team members has led to encouraging results. Careful allograft monitoring was instituted with the use of routine allograft skin biopsy and mucosal biopsy. If clinical rejection was suspected, biopsy samples were also obtained.

Our experience using skin and mucosal biopsy is consistent with much of the world experience. Histological evidence of nonspecific mucosal inflammation is seen in both routine biopsies (Fig. 1) and episodes of clinical rejection. Similar to previous published studies, mucosal inflammation was present without clinical signs of rejection. However, we continue to use both types of biopsies to monitor for rejection. In general, isolated inflammation of mucosa or skin is considered subclinical rejection and not treated.

\section{Antibody Mediated Rejection and Chronic Rejection}

To date only one case of antibody mediated rejection (AMR) has been described in VCA including limb or facial transplantation [40]. Despite C4d being implicated in AMR reported in renal allograft biopsies, the clinical experience of $\mathrm{C} 4 \mathrm{~d}$ deposits in facial transplant biopsies does not always support evidence of rejection. In fact, multiple hand transplants without clinical signs of rejection have been reported to have $\mathrm{C} 4 \mathrm{~d}$ deposition [41,42] yet other limb and face allografts report no such findings of $\mathrm{C} 4 \mathrm{~d}$ deposition on histological assessment [43•]. Patients with donor specific antibodies after receiving a hand transplant did not correspond with $\mathrm{C} 4 \mathrm{~d}$ deposition on biopsy [44]. Complicating matters further, a rat VCA model has recently shown that rats sensitized with donor specific antibodies exhibit accelerated rejection, but the response is not hyperacute as expected [45]. The underlying mechanisms of antibody mediated rejection in VCA remain poorly understood and are not well-defined.

Chronic rejection has yet to be reported in facial VCA, and only two patients have manifested signs suggestive of chronic rejection in limb transplantation [46, 47]. Despite the increased rates of acute rejection, chronic rejection is extremely rare. In nonhuman primates, Mundinger et al. elucidated that chronic allograft rejection was an entity detectable with neointimal proliferation, transplant vasculopathy, vessel wall fibrosis, progressive luminal occlusion, and detection of tertiary lymphoid follicles [48]. However, in human facial VCA chronic rejection has not yet been identified. Chronic rejection has been observed in hand transplantation. The first hand transplant in the United States developed acute arterial thrombosis 275 days after transplantation and was reported to have aggressive intimal hyperplasia [47]. Prior to the acute episode

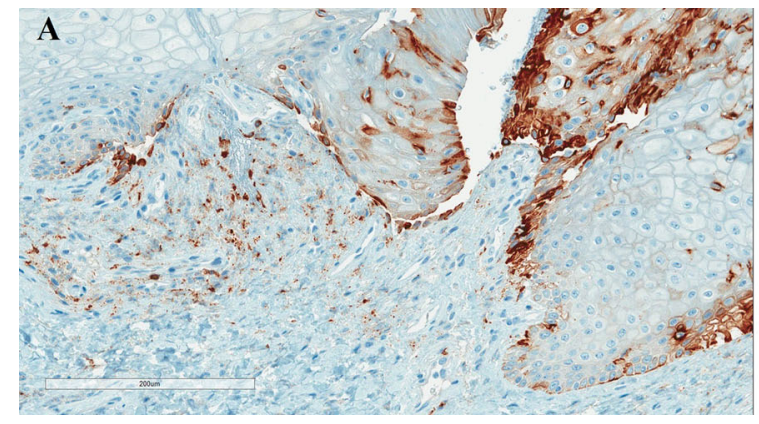

Fig. 1 Histological specimen of CD8 stained (A) oral mucosa and (B) allograft skin on routine scheduled biopsy on postoperative day 51 . The skin and mucosa appeared normal on visual examination. T-cell infiltrates

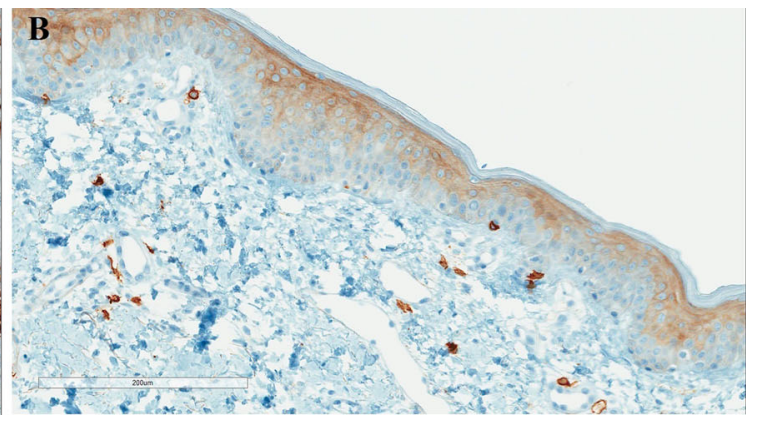

of unclear significance are noted in both the mucosal and skin biopsy, however, the changes are more pronounced in the mucosal sample. As there was no clinical evidence of rejection, the patient was not treated 
of ischemia, no skin lesions were observed and skin biopsies did not reveal evidence of rejection. Recent histology, magnetic resonance imaging, ultrasonography, and high resolution computerized tomography scans were reviewed to evaluate for evidence of chronic rejection including dermal fibrosis and vascular stenosis in facial VCA [43•]. Absence of dermal fibrosis and intimal hyperplasia continue to support the notion that there is currently no evidence of chronic rejection in facial vascularized composite allotransplantation.

\section{Conclusion}

The methods of monitoring facial allograft function are largely accomplished with clinical examination and confirmation with skin biopsy. Acute rejection can manifest as a focal skin lesion or diffusely with varying intensity. Close clinical follow-up and employing routine skin and mucosal biopsies allow for detection and treatment of early acute rejection and may provide insight into active and resolving rejection, as well as about nonalloimmune related pathological processes.

Acknowledgments Eduardo D. Rodriguez is supported by the Office of Naval Research Grant N00014-10-1-0868.

\section{Compliance with Ethics Guidelines}

Conflict of Interest Michael Sosin, Jhade D. Woodall, Benjamin D. Schultz, Arif Chaudhry, Branko Bojovic, Michael R. Christy, and Cinthia B. Drachenberg declare that they have no conflict of interest.

Eduardo D. Rodriguez has received grants, payments for lectures, travel accommodations, and expenses for unrelated activities from DePuy Synthes CMF. He has also received educational grant support and speaker honorarium from KLS Martin.

Human and Animal Rights and Informed Consent This article does not contain any studies with human or animal subjects performed by any of the authors.

\section{References}

Papers of particular interest, published recently, have been highlighted as:

- Of importance

-. Of major importance

1. Khalifian S, Brazio PS, Mohan R, Shaffer C, Brandacher G, Barth RN, et al. Facial Transplantation: the first 9 years. Lancet. 2014.

2. Kaufman CL, Ouseph R, Marvin MR, Manon-Matos Y, Blair B, Kutz JE. Monitoring and long-term outcomes in vascularized composite allotransplantation. Curr Opin Organ Transplant. 2013;18: 652-8.

3. Pomahac B, Gobble RM, Schneeberger S. Facial and hand allotransplantation. Cold Spring Harb Perspect Med. 2014;4.

4. Dubernard JM, Owen ER, Herzberg G, et al. Human hand allograft: report on first 6 months. Lancet. 1999;353:1315-20.
5. Devauchelle B, Badet L, Lengelé B, Morelon E, Testelin S, Michallet M, et al. First human face allograft: early report. Lancet. 2006;368:203-9.

6. Dorafshar AH, Bojovic B, Christy MR, Borsuk DE, Iliff NT, Brown EN, et al. Total face, double jaw, and tongue transplantation: an evolutionary concept. Plast Reconstr Surg. 2013;131:241-51.

7. Lee WP, Yaremchuk MJ, Pan YC, Randolph MA, Tan CM, Weiland AJ. Relative antigenicity of components of a vascularized limb allograft. Plast Reconstr Surg. 1991;87:401-11.

8. Cendales LC, Kanitakis J, Schneeberger S, Burns C, Ruiz P, Landin L, et al. The Banff 2007 working classification of skin-containing composite tissue allograft pathology. Am J Transplant. 2008;8: 1396-400.

9. Khalifian S, Broyles JM, Tuffaha SH, Alrakan M, Ibrahim Z, Sarhane KA. Immune mechanisms of ischemia-reperfusion injury in transplantation. Open J Immunol. 2013;3:158-64.

10. Van der Touw W, Cravedi P, Kwan W, Paz-Artal E, Merad M, Heeger PS. Cutting edge: receptors for $\mathrm{C} 3 \mathrm{a}$ and $\mathrm{C} 5 \mathrm{a}$ modulate stability of alloantigen-reactive induced regulatory $\mathrm{T}$ cells. J Immunol. 2013;190:5921-5.

11. Sarhane KA, Tuffaha SH, Broyles JM, Ibrahim AE, Khalifian S, Baltodano $\mathrm{P}$, et al. A critical analysis of rejection in vascularized composite allotransplantation: clinical, cellular and molecular aspects, current challenges, and novel concepts. Front Immunol. 2013;25(4):406.

12. Morelon E, Kanitakis J, Petruzzo P. Immunological issues in clinical composite tissue allotransplantation: where do we stand today? Transplantation. 2012;93:855-9.

13. Murphy BD, Zuker RM, Borschel GH. Vascularized composite allotransplantation: an update on medical and surgical progress and remaining challenges. J Plast Reconstr Aesthet Surg. 2013;66:1449-55.

14. Gaspari AA, Katz SI. Induction and functional characterization of class II MHC (Ia) antigens on murine keratinocytes. J Immunol. 1988;140:2956-63.

15. Sugita K, Kabashima K, Atarashi K, et al. Innate immunity mediated by epidermal keratinocytes promotes acquired immunity involving Langerhans cells and T cells in the skin. Clin Exp Immunol. 2007;147:176-83.

16. Uchi H, Terao H, Koga T, et al. Cytokines and chemokines in the epidermis. J Dermatol Sci. 2000;24 suppl 1:29-38.

17. Schneeberger S, Khalifian S, Brandacher G. Immunosuppression and monitoring of rejection in hand transplantation. Tech Hand Upper Extrem Surg. 2013;17:208-14.

18. Kanitakis J, Badet L, Petruzzo P, Béziat JL, Morelon E, Lefrançois $\mathrm{N}$, et al. Clinicopathologic monitoring of the skin and oral mucosa of the first human face allograft: report on the first eight months. Transplantation. 2006;82:1610-5.

19. Dubernard JM, Lengelé B, Morelon E, Testelin S, Badet L, Moure $\mathrm{C}$, et al. Outcomes 18 months after the first human partial face transplantation. N Engl J Med. 2007;357:2451-60.

20. Pomahac B, Pribaz J, Eriksson E, Annino D, Caterson S, Sampson $\mathrm{C}$, et al. Restoration of facial form and function after severe disfigurement from burn injury by a composite facial allograft. Am J Transplant. 2011;11:386-93.

21. Morelon E, Testelin S, Petruzzo P, Badet L, Michallet M, Dubois V, et al. Face transplantation with combined hematopoietic stem cell infusion and vascularized bone marrow: first year of follow-up. Transplant Int. 2011;24 Suppl 2:74.

22.• Pomahac B, Pribaz J, Eriksson E, Bueno EM, Diaz-Siso JR, Rybicki FJ, et al. Three patients with full facial transplantation. N Engl J Med. 2012;366:715-22. Review of three patients who received face transplantations with a consistent protocol for immunosuppression with reported outcomes six months postoperatively.

23.• Bergfeld W, Klimczak A, Stratton JS, Siemionow MZ. A four-year pathology review of the near total face transplant. Am J Transplant. 
2013;13:2750-64. A comprehensive 4-year review of the skin and mucosal biopsy results from a partial face transplantation, and the need for a classification system that is able to address the different presentations in skin and mucosa during acute rejection.

24. Siemionow M, Gharb BB, Rampazzo A. Successes and lessons learned after more than a decade of upper extremity and face transplantation. Curr Opin Organ Transplant. 2013;18:633-9.

25. Siemionow M, Ozturk C. Face transplantation: outcomes, concerns, controversies, and future directions. J Craniofac Surg. 2012;23: 254-9.

26. Schneeberger S, Lucchina S, Lanzetta M, Brandacher G, Bösmüller C, Steurer W, et al. Cytomegalovirus-related complications in human hand transplantation. Transplantation. 2005;80:441-7.

27. Erdbruegger U, Scheffner I, Mengel M, et al. Impact of CMV infection on acute rejection and long-term renal allograft function: a systematic analysis in patients with protocol biopsies and indicated biopsies. Nephrol Dial Transplant. 2012;27:435-43.

28. Dzabic M, Rahbar A, Yaiw KC, et al. Intragraft cytomegalovirus protein expression is associated with reduced renal allograft survival. Clin Infect Dis. 2011;53:969-76.

29. Lantieri L, Meningaud JP, Grimbert P, Bellivier F, Lefaucheur JP, Ortonne N, et al. Repair of the lower and middle parts of the face by composite tissue allotransplantation in a patient withmassive plexiform neurofibroma: a 1-year follow-up study. Lancet. 2008;372: $639-45$.

30. Siemionow M, Papay F, Alam D, Bernard S, Djohan R, Gordon C, et al. Near-total human face transplantation for a severely disfigured patient in the USA. Lancet. 2009;374:203-9.

31. Lantieri L, Hivelin M, Audard V, Benjoar MD, Meningaud JP, Bellivier F, et al. Feasibility, reproducibility, risks and benefits of face transplantation: a prospective study of outcomes. Am J Transplant. 2011;11:367-78.

32. BenMarzouk-Hidalgo OJ, Cordero E, Gómez-Cía T, Sánchez M, González-Padilla JD, Infante-Cossio P, et al. First face compositetissue transplant recipient successfully treated for cytomegalovirus infection with preemptive valganciclovir treatment. Antimicrob Agents Chemother. 2011;55:5949-51.

33. Barret JP, Gavaldà J, Bueno J, Nuvials X, Pont $\mathrm{T}$, Masnou N, et al. Full face transplant: the first case report. Ann Surg. 2011;254:252-6.

34. Petruzzo P, Testelin S, Kanitakis J, Badet L, Lengelé B, Girbon JP, et al. First human face transplantation: 5 years outcomes. Transplantation. 2012;93:236-40.

35. Carty MJ, Hivelin M, Dumontier C, Talbot SG, Benjoar MD, Pribaz $\mathrm{JJ}$, et al. Lessons learned from simultaneous face and bilateral hand allotransplantation. Plast Reconstr Surg. 2013;132:423-32.
36. Papadimitriou JC, Cangro CB, Lustberg A, et al. Histologic featuresof mycophenolate mofetil-related colitis: a graft-versushost disease-like pattern. Int J Surg Pathol. 2003;11:295-302.

37. Nguyen T, Park JY, Scudiere JR, Montgomery E. Mycophenolic acid (cellcept and myofortic) induced injury of the upper GI tract. Am J Surg Pathol. 2009;33:1355-63.

38. van Gelder T, ter Meulen CG, Hené R, Weimar W, Hoitsma A. Oral ulcers in kidney transplant recipients treated with sirolimus and mycophenolate mofetil. Transplantation. 2003;75:788-91.

39. Campistol JM, de Fijter JW, Flechner SM, Langone A, Morelon E, Stockfleth E. mTOR inhibitor-associated dermatologic and mucosal problems. Clin Transplant. 2010;24:149-56.

40. Chandraker A, Arscott R, Murphy GF, Lian CG, Bueno EM, Marty FM, et al. The management of antibody-mediated rejection in the first presensitized recipient of a full-face allotransplant. Am J Transplant. 2014.

41. Hautz T, Zelger B, Brandacher G, Mueller H, Grahammer J, Zelger $\mathrm{B}$, et al. Histopathologic characterization of mild rejection (grade I) in skin biopsies of human hand allografts. Transpl Int. 2012;25:56-63.

42. Landin L, Cavadas PC, Ibanez J, Roger I, Vera-Sempere F. CD3+mediated rejection and $\mathrm{C} 4 \mathrm{~d}$ deposition in two composite tissue (bilateral hand) allograft recipients after induction with alemtuzumab. Transplantation. 2009;87:776.

43. Petruzzo P, Kanitakis J, Badet L, Pialat JB, Boutroy S, Charpulat R, et al. Long-term follow-up in composite tissue allotransplantation: in-depth study of five (hand and face) recipients. Am J Transplant. 2011;11:808-16. A review of five individuals who received bilateral hand or face transplants with at least two years of follow-up without evidence of chronic rejection.

44. Brandacher G. Composite tissue transplantation. Methods Mol Biol. 2013;1034:103-15.

45. Wu S, Xu H, Chen B, Wen Y, Ikusika OM, Ocker A. Sensitized recipients exhibit accelerated but not hyperacute rejection of vascularized composite tissue allografts. Transplantation. 2011;92: 627-33.

46. Pei G, Xiang D, Gu L, Wang G, Zhu L, Yu L, et al. A report of 15 hand allotransplantations in 12 patients and their outcomes in China. Transplantation. 2012;94:1052-9.

47. Kaufman CL, Ouseph R, Blair B, Kutz JE, Tsai TM, Scheker LR, et al. Graft vasculopathy in clinical hand transplantation. Am J Transplant. 2012;12:1004-16.

48. Mundinger GS, Munivenkatappa R, Drachenberg CB, Ha JS, Vaca EE, Shipley ST, et al. Histopathology of chronic rejection in a nonhuman primate model of vascularized composite allotransplantation. Transplantation. 2013;95:1204-10. 